\title{
Presence of Periampullary Diverticulum Is Not a Hurdle to Successful Endoscopic Retrograde Cholangiopancreatography
}

\author{
Jimin Han \\ Department of Internal Medicine, Catholic University of Daegu School of Medicine, Daegu, Korea
}

See "Impact of Periampullary Diverticulum on ERCP Performance: A Matched Case-Control Study" by Juan E. Corral, Omar Y. Mousa, Paul T. Kröner, et al., on page 65-71.

In this issue of Clinical Endoscopy, Corral et al. compared technical success of endoscopic retrograde cholangiopancreatography (ERCP) between patients with periampullary diverticulum (PAD) and age- and gender-matched controls using the Clinical Outcomes Research Initiative (CORI) database. ${ }^{1}$

The presence of PAD has been associated with the recurrence of bile duct stone, gallstones, and cholangitis. ${ }^{2,3}$ However, previous studies on its effect on successful ERCP have shown conflicting results. Vaira et al. reported that success rates of selective cannulation and endoscopic sphincterotomy were significantly higher in the patients without PAD than in the patients with PAD (96.7\% vs. $94.2 \%, p<0.05$; $98.0 \%$ vs. $95.2 \%$, $p<0.05) .{ }^{4}$ Bile duct stone clearance rate was not significantly different between two groups. ${ }^{4}$ On the other hand, Chen et al. showed that success rate of selective cannulation was similar between the patients without PAD and patients with PAD (99.07\% vs. 98.59\%, $p=0.225)^{3}$. Bile duct stone clearance rate after sphincterotomy was lower in the patients with PAD (83.53\% vs. $94.31 \%, p=0.005){ }^{3}$ In this issue of Clinical Endos$\operatorname{cop} y$, Corral et al. demonstrated that there was no significant difference in technical success rate of ERCP between patients

Received: December 5, 2018 Revised: December 7, 2018

Accepted: December 8, 2018

Correspondence: Jimin Han

Department of Internal Medicine, Catholic University of Daegu School of Medicine, 33 Duryugongwon-ro 17-gil, Nam-gu, Daegu 42472, Korea

Tel: +82-53-650-3442, Fax: +82-53-621-4487, E-mail: jmhan@cu.ac.kr ORCID: https://orcid.org/0000-0001-8674-370X

(c) This is an Open Access article distributed under the terms of the Creative Commons Attribution Non-Commercial License (http://creativecommons.org/ licenses/by-nc/3.0) which permits unrestricted non-commercial use, distribution, and reproduction in any medium, provided the original work is properly cited. without PAD and patients with PAD (96.0\% vs. 94.9\%, $p=0.2){ }^{1}$ In the patients with $\mathrm{PAD}$, the procedure time was shorter, but the fluoroscopy time was similar to that for patients without PAD. ${ }^{1}$ Moreover, when the patients with gallstone but without PAD were compared to those with both PAD and gallstone, technical success rate of ERCP was still similar (99.2\% vs. 99.1\%, $p=0.8)^{1}$.

In addition to this being a multi-centric study with the largest number of patients $(n=1,089)$ to evaluate the impact of PAD on ERCP, the use of the CORI database is also noteworthy. Use of the CORI database resulted in representation of full spectrum of gastroenterology practices in the United States. It was established in 1995 under the auspice of the American Society for Gastrointestinal Endoscopy to evaluate clinical outcomes of gastrointestinal endoscopic procedures. ${ }^{5}$ Its primary objectives are to study the outcome of gastrointestinal endoscopic procedures in various clinical settings and to examine endoscopic practice patterns. ${ }^{5}$ Current number of participating sites is 70, including hospitals, ambulatory care centers, private practices, universities, and Veteran's hospitals. ${ }^{5}$ The collected data contain no patient identifier and are merged and stored in the National Endoscopic Database. ${ }^{5}$

However, limitations of this study stems from many caveats of the CORI database itself. ${ }^{5}$ Because the CORI database is a clinical database, not an analytical data set, a great deal of information is not documented. For example, there is no information on type of $\mathrm{PAD}$, stone size, anatomic location, or degree of bile duct dilatation. Although duplicate patient identifier had been eliminated in this study, it cannot be ensured that the patient had not undergone ERCP previously at sites 
not participating in the CORI database. When interpreting the results, one should be aware that the participating sites are not representative of all gastrointestinal practices in the United States; veteran's hospitals and academic medical centers may be over-represented. Because only immediate complications are recorded in the CORI database, and therefore, overall procedure-related complications were not included in the current study design, impact of PAD on ERCP-related complications could not be analyzed. It would be also worthwhile to develop a prospective multi-centric endoscopic database in other country.

PAD is a common incidental finding during ERCP; it is found in $4.7 \%$ of ERCP cases and in $8.6 \%$ of patients who are older than 70 years. ${ }^{1}$ Its prevalence may be higher in patients with bile duct stones. ${ }^{2,3}$ Whenever an endoscopist encounters a PAD during ERCP, one should remember the findings of the current study and proceed with confidence. Even if it looks daunting, the presence of PAD is not a hurdle to successful ERCP.
Conflicts of Interest

The author has no financial conflicts of interest.

\section{REFERENCES}

1. Corral JE, Mousa OY, Kröner PT, Gomez V, Lukens FJ. Impact of periampullary diverticulum on ERCP performance: a matched case-control study. Clin Endosc 2019;52:65-71.

2. Kim KY, Han J, Kim HG, et al. Late complications and stone recurrence rates after bile duct stone removal by endoscopic sphincterotomy and large balloon dilation are similar to those after endoscopic sphincterotomy alone. Clin Endosc 2013;46:637-642.

3. Chen L, Xia L, Lu Y, Bie L, Gong B. Influence of periampullary diverticulum on the occurrence of pancreaticobiliary diseases and outcomes of endoscopic retrograde cholangiopancreatography. Eur J Gastroenterol Hepatol 2017;29:105-111.

4. Vaira D, Dowsett JF, Hatfield AR, et al. Is duodenal diverticulum a risk factor for sphincterotomy? Gut 1989;30:939-942.

5. National Institute of Diabetes and Digestive and Kidney Diseases. Clinical outcomes research initiative (CORI) [Internet]. Bethesda (MD): National Institutes of Health; c2016 [cited 2018 Dec 7]. Available from: https://repository.niddk.nih.gov/studies/cori/. 